\title{
CORRECTIONS
}

\section{Correction 1}

Cite as: Can Urol Assoc J 2014;8(3-4):84. http://dx.doi.org/10.5489/cuai.2091

Published online April 14, 2014.

$\mathrm{n}$ the following article published in the March online issue of $C U A J$, the following correction is noted in the author affiliations.

\section{Dong Soo Park, MD; Jin Ho Hwang, MD; Moon Hyung Kang, MD; Jong Jin Oh, MD}

*Department of Urology, CHA Bundang Medical Center, CHA University, Bundang-gu, Seongnam-si, Korea; 'Department of Urology, Seoul National University Bundang Hospital, Bundang-gu, Seongnamsi, Korea

\section{Reference}

1. Park DS, Hwang JH, Kang MH, et al. Association between R.E.N.A.L. nephrometry score and perioperative outcomes following open partial nephrectomy under cold ischemia. Can Urol Assoc J 2014;8:e137-41. http://dx.doi.org/10.5489/cuaj.1372

\section{Correction 2}

Cite as: Can Urol Assoc J 2014;8(3-4):84. http://dx.doi.org/10.5489/cuaj.2092 Published online April 14, 2014.

$\mathrm{n}$ the article entitled "Comparison of outpatient versus inpatient transurethral prostate resection for benign prostatic hyperplasia: Comparative, prospective bi-centre study, ${ }^{\prime 1}$ there are corrections to the author order and affiliations.

The correct order and affiliations are as follows:

Hoon Choi, MD; '* Jae Heon Kim, MD; ' Jae Young Park, MD; ${ }^{*}$ Ji Sung Shim, MD; ; Jeong Gu Lee, MD; Du Geon Moon, MD;s Jeong woo Yoo, MD;* Jae Hyun Bae, MD*

*Department of Urology, Korea University Ansan Hospital, Korea University College of Medicine, Danwon-gu, Ansan, Korea; 'Department of Urology, Soonchunhyang University College of Medicine, Seoul, Korea; ${ }^{\circledR}$ Department of Urology, Korea University College of Medicine, Seoul , Korea; ${ }^{*}$ Tower Urology Clinic, Nambusunhwan-ro, Gangnam-gu, Seoul, Korea

\section{Reference}

1. Kim JH, Park JY, Shim JS, et al. Comparison of outpatient versus inpatient transurethral prostate resection for benign prostatic hyperplasia: Comparative, prospective bi-centre study. Can Urol Assoc J 2014;8:e30-5. http://dx.doi.org/10.5489/cuaj.1370 